\title{
Structural Properties of MnTe, ZnTe, and ZnMnTe
}

\author{
N. Gonzalez Szwacki, E. Przeździecka, E. Dynowska, \\ P. BogusŁawski and J. Kossut \\ Institute of Physics, Polish Academy of Sciences \\ al. Lotników 32/46, 02-668 Warsaw, Poland
}

\begin{abstract}
Structural properties of $\mathrm{ZnTe}$, MnTe, and $\mathrm{Mn}_{1-x} \mathrm{Zn}_{x}$ Te alloy with zinc-blende, NiAs, and wurtzite phases were investigated by ab initio calculations. The calculated structural properties are in good agreement with the available experimental data. Theory predicts that the zinc-blende phase is more stable than wurtzite for all compositions. $\mathrm{Mn}_{1-x} \mathrm{Zn}_{x}$ Te samples with $0.01<x<0.20$ were grown by MBE. X-ray analysis of their crystalline structure revealed the presence of zinc-blende, wurtzite, and NiAs phases. The dominant phase changes from NiAs for the sample with $x=0.01$ to wurtzite for $x=0.20$. The observed stabilization of the wurtzite phase is possibly due to the hexagonal structure of the MnTe buffer.
\end{abstract}

PACS numbers: 61.50.Ah, 75.50.Pp, 61.10.-i

\section{Introduction}

Mn-doped IIB-VI semimagnetic semiconductors have attracted considerable interest for several years [1]. Beside the well-known changes in the electronic structure of ordinary ternary semiconducting compounds due to the composition, IIB-VI compounds show novel magneto-optical and magnetotransport properties if the cation is partly substituted by Mn with its half-filled $3 d$ shell [1]. There are no previous reports on theoretical and experimental studies of Zn-doped MnTe semiconductor. This material could be of special interest in the construction of room temperature antiferromagnetic alloys.

Here we present $a b$ initio calculations based on a plane-wave pseudopotential method within the local spin density approximation (LSDA) to investigate phase stability and structural and elastic properties of ZnTe, MnTe, and ZnMnTe in zinc-blende (ZB), NiAs, and wurtzite (W) phases. The cut-off energy of 35 Ry 
for the plane-wave basis set was sufficient to obtain convergent results. In the pseudopotential description, occupied $d$ orbitals of $\mathrm{Zn}$ and $\mathrm{Mn}$ atoms were treated as valence states. Calculations in this work have been done using the PWscf package [2].

\section{Experiment}

Five samples were grown using two-chambers MBE EPI 620 system. A $10 \mathrm{kV}$ RHEED analyzer was used to control the two-dimensional character of the growth process. The growth was performed on $10 \times 10 \mathrm{~mm}^{2} \mathrm{Al}_{2} \mathrm{O}_{3}$ (0001)-oriented hexagonal substrates covered with MnTe buffer layers of the thickness of $100 \mathrm{~nm}$. The $\mathrm{Mn}_{1-x} \mathrm{Zn}_{x}$ Te overlayers of the thickness of $2 \mu \mathrm{m}$ have been grown with the $\mathrm{Zn}$ content varying from about 1 to about 20 atomic percent. The Zn content has been changed by changing the temperature of the $\mathrm{Zn}$ effusion cell, and the alloy composition was estimated by X-ray fluorescence analysis. Further details will be published elsewhere [3]. The post-growth structural characterization was made by means of X-ray diffraction. The data were collected at an X'Pert-MPD Bragg-Brentano diffractometer equipped with a copper X-ray tube, a focusing incident-beam monochromator, and a semiconductor strip detector. The applied instrument allows us to obtain diffraction patterns with excellent resolution $\left(0.04^{\circ}\right.$ in $2 \theta$ units). For all five compositions the powder patterns are composed of strong lines demonstrating the coexistence of $\mathrm{W}, \mathrm{ZB}$, and NiAs-type $\mathrm{Mn}_{1-x} \mathrm{Zn}_{x}$ Te. The (0002) wurtzite and (111) ZB lines are very close to each other, but they were resolved due to high resolution of the apparatus. The preferred orientation of NiAstype and $\mathrm{W}$ phases is (0001), while in the case of sphalerite it is (111). As follows

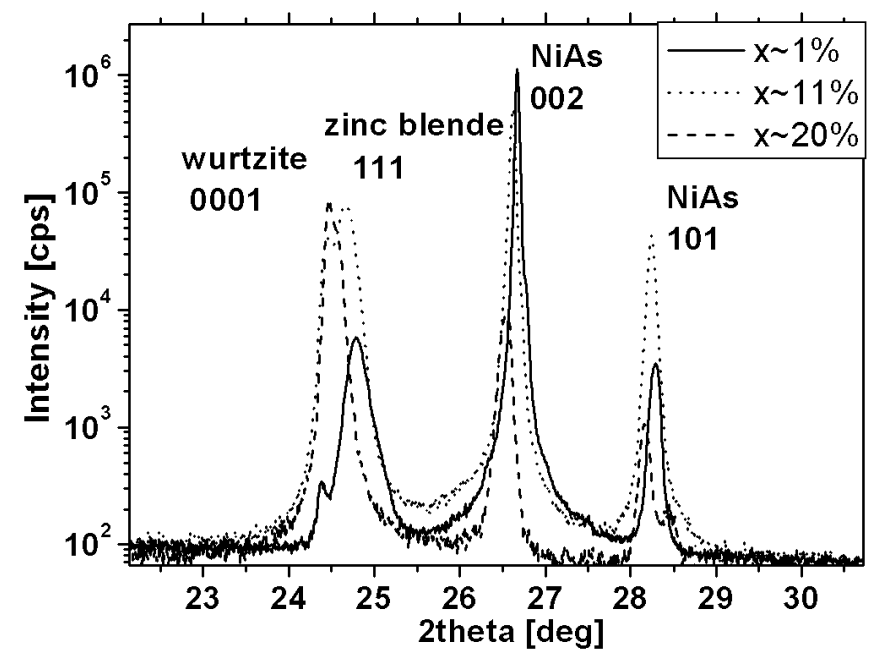

Fig. 1. X-ray diffraction intensity for $\mathrm{Mn}_{1-x} \mathrm{Zn}_{x}$ Te with $x=0.01,0.11$, and 0.20 . The reflexes from the $\mathrm{W}, \mathrm{ZB}$, and NiAs structures are indicated. 
from Fig. 1, the relative contributions of these phases depend on the $\mathrm{Zn}$ content: for the smallest $\mathrm{Zn}$ content the NiAs phase dominates, while with increasing $\mathrm{Zn}$ content the relative volume of $\mathrm{W}$ and $\mathrm{ZB}$ phases increases as compared with that of NiAs. In the sample with nominally the highest $\mathrm{Zn}$ content the $\mathrm{W}$ phase is dominant.

\section{Lattice description}

We present in Fig. 2a-c unit cells in the zinc-blende, wurtzite, and NiAs structures, respectively. The elementary cells of the wurtzite and the NiAs structures are three times smaller than these presented in Fig. 2b and c, and there are three $(a, c$, and $u$ ) and two $(a$ and $c$ ) parameters describing these unit cells, respectively. The elementary unit cell of the NiAs structure is shown in Fig. 2d. The zinc-blende structure has two cubic close packed (face centered) sublattices of anion and cation atoms, respectively. Atoms of one sublattice occupy tetrahedral interstitial sites of the second sublattice, giving a layer stacking along (111) that exhibits an ABCABC... sequence. The wurtzite structure consists of two hexagonal close packed (hcp) sublattices, where the anion (cation) sublattice is

(a)

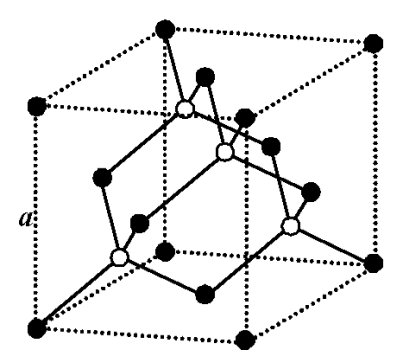

(b)

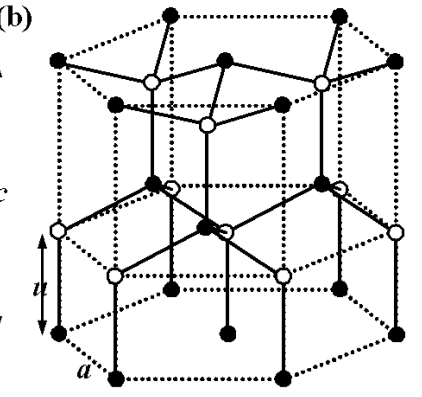

(e)

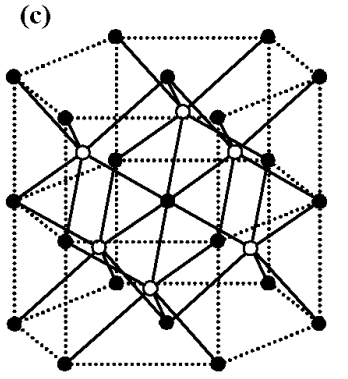

(d)
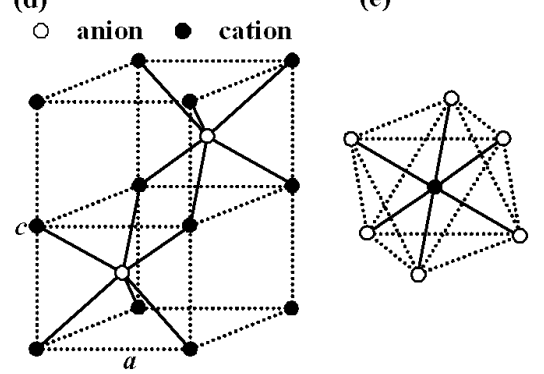

Fig. 2. Unit cells in the zinc-blende (a), wurtzite (b), and NiAs structure (c). For NiAs we also show the elementary unit cell (d) and the octahedral neigborhood of cation atoms (e). 
located above the cation (anion) sublattice at a distance $u$ (see Fig. 2b). The stacking sequence of the layers in both sublattices is ABAB..., and the atoms of one sublattice are located in the tetrahedral interstices of the other. In contrast, the NiAs structure can be viewed as an hcp anion sublattice with cation atoms in the octahedral interstices (the tetrahedral interstices, occupied in the $\mathrm{W}$ structure, are vacant). In fact, the cation atoms in the consecutive (0001) layers are located directly on top of each other (see Fig. 2c and d), so that the stacking sequence is AAA... and the cations do not form an hcp sublattice. The main difference between these three structures is that in the NiAs structure each atom has six nearest neighbors, while in both the $\mathrm{ZB}$ and $\mathrm{W}$ structures each atom has a tetrahedral neighborhood. Indeed, in Fig. 2e we can see that in the NiAs structure a cation is surrounded by six anions forming an octahedron, while an anion is surrounded by six cation atoms forming a trigonal prism, see e.g. Fig. 2d.

\section{Structural properties and phase stability of studied systems}

To investigate the structural properties and phase stability of all systems we calculate the total energy $\left(E_{\text {tot }}\right)$ of these systems as a function of lattice constants. For each crystal, both the equilibrium lattice parameters and ground state energies were obtained by fitting the data points to polynomial curves. For the hexagonal phases of binary compounds and all phases of the ternary compound the structural relaxations were taken into account. In agreement with experiment [4] we find that the stable phase of ZnTe is $\mathrm{ZB}$, lower in energy by $7 \mathrm{meV} /$ atom than the W phase. For this crystal, the NiAs structure is highly unfavorable, being higher in energy by $250 \mathrm{meV}$ /atom. The structural similarities between $\mathrm{W}$ and $\mathrm{ZB}$ and the small differences in the total energies are manifested by the well-known W-ZB polytypism: depending on the details of the growth parameters, a number

TABLE

Comparison of calculated values for equilibrium lattice constants, bulk modulus $B$, and pressure coefficients $B^{\prime}$ with experimental data.

\begin{tabular}{c|c|c|c|c}
\hline \hline & $a[\AA]$ & $c / a$ & $B[\mathrm{GPa}]$ & $B^{\prime}$ \\
\hline \multicolumn{5}{c}{ ZnTe (ZB) } \\
\hline LSDA & 6.042 & & 55.0 & 4.3 \\
EXP & $6.100^{a}$ & & $52.8^{a}$ & \\
\hline \multicolumn{5}{c}{ MnTe (NiAs) } \\
\hline LSDA & 3.919 & 1.606 & 41.3 & 2.0 \\
EXP & $4.158^{b}$ & $1.618^{b}$ & & \\
\hline
\end{tabular}

${ }^{a}$ Ref. [7]; ${ }^{b}$ Ref. [8] 


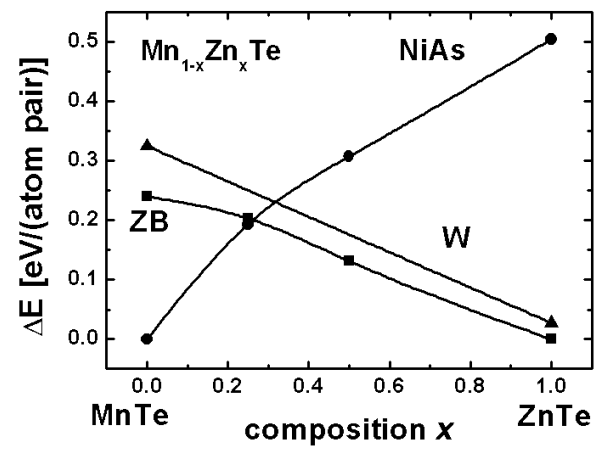

Fig. 3. Total energy difference $\Delta E$ versus composition $x$ for $\mathrm{Mn}_{1-x} \mathrm{Zn}_{x}$ Te in three phases: ZB, W, and NiAs.

of binary semiconductors ( $\mathrm{SiC}, \mathrm{CdS}, \mathrm{CdSe}, \mathrm{ZnS}, \mathrm{CuCl}$, and $\mathrm{CuBr}$ ) can be prepared at ambient pressure in either forms [5]. On the other hand, the stable structure of the magnetic semiconductor MnTe is NiAs, which is lower in energy than the ZB and the $\mathrm{W}$ phases by 100 and $140 \mathrm{meV} /$ atom, respectively. In the calculations we have considered ferromagnetic (FM) as well as antiferromagnetic (AF) ordering of the magnetic moments of Mn. The AF ordering of type-I was assumed for all studied structures. In agreement with experiment [6] the AF ordering is more stable than FM in all cases.

The bulk properties of the stable phases of binary compounds are summarized in Table, and compared with experimental data [7, 8]. As one can see, we have obtained an agreement with experimental values that is better for ZnTe than for MnTe, but satisfactory for both crystals.

Finally, we describe the structural stability of $\mathrm{Mn}_{1-x} \mathrm{Zn}_{x}$ Te alloy. Figure 3 shows the total energy difference

$$
\Delta E_{\alpha}(x)=E_{\alpha}^{\mathrm{Mn}_{1-x} \mathrm{Zn}_{x} \mathrm{Te}}-(1-x) E_{\mathrm{NiAs}}^{\mathrm{MnTe}}-x E_{\mathrm{ZB}}^{\mathrm{ZnTe}}
$$

as a function of the composition $x$ for all three phases ( $\alpha=\mathrm{W}, \mathrm{ZB}$, and NiAs). From the figure we can see that the $\mathrm{W}$ phase is unstable for all alloy compositions. The stability of the NiAs phase increases and of the ZB phase decreases with increasing $x$. There is a crossover between the $\mathrm{ZB}$ and the NiAs curves for $x \approx 0.25$.

\section{Summary}

We have studied structural properties of $\mathrm{ZnTe}, \mathrm{MnTe}$, and $\mathrm{Mn}_{1-x} \mathrm{Zn}_{x} \mathrm{Te}$ alloy with ZB, NiAs, and $\mathrm{W}$ phases by ab initio calculations. The calculated lattice parameters are in good agreement with the available experimental data. Theory predicts that the dominant phases for $x<0.25$ and $x>0.25$ are NiAs and $\mathrm{ZB}$, respectively, and that the $\mathrm{W}$ phase is higher in energy than $\mathrm{ZB}$ by $0.04 \mathrm{eV}$ for all compositions. $\mathrm{Mn}_{1-x} \mathrm{Zn}_{x}$ Te samples with $0.01<x<0.20$ were grown by 
MBE, and their structure was analyzed by X-ray diffraction. The co-existence of ZB, W, and NiAs phases was detected. The dominant phase changes from NiAs for the sample with $x=0.01$ to W for $x=0.20$. The observed stabilization of the $\mathrm{W}$ phase is possibly driven by the hexagonal NiAs structure of the MnTe buffer and its orientation, as well as the small energy difference between the ZB and W structures.

\section{Acknowledgments}

The research was supported by grant from the State Committee for Scientific Research PBZ-KBN-044/P03/2001, and grant "Nosce Memorias" from the European Commission. J. Kossut and E. Przeździecka acknowledge the support from Foundation for Polish Science.

\section{References}

[1] R. Weidemann, H.-E. Gumlich, M. Kupsch, H.-U. Middelmann, Phys. Rev. B 45, 1172 (1992).

[2] S. Baroni, A. Dal Corso, S. de Gironcoli, P. Giannozzi, http://www.pwscf.org.

[3] E. Dynowska, E. Przeździecka, J. Kossut, N. Gonzalez Szwacki, P. Bogusławski, to be published.

[4] Physics of II-VI and I-VII Compounds, Semimagnetic Semiconductors, Vol. 17 of Landolt-Börnstein New Series, Ed. K.-H. Hellwege, Springer-Verlag, Berlin 1982.

[5] Chin-Yu Yeh, Z.W. Lu, S. Froyen, A. Zunger, Phys. Rev. B 46, 10086 (1992).

[6] T.M. Giebultowicz, P. Kłosowski, N. Samarth, H. Luo, J.K. Furdyna, J.J. Rhyne, Phys. Rev. B 48, 12817 (1993).

[7] Numerical Data and Functional Relationships in Science and Technology, Group III, Vol. 22a of Landolt-Börnstein New Series, Eds. K.-H. Hellwege, O. Madelung, Springer-Verlag, New York 1982.

[8] E. Przeździecka, J. Kossut, to be published. 